\title{
Seismically active area monitoring by robust TIR satellite techniques: a sensitivity analysis on low magnitude earthquakes in Greece and Turkey
}

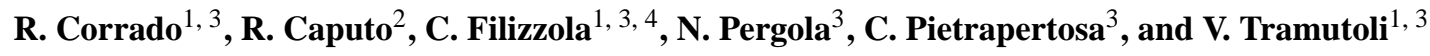 \\ ${ }^{1}$ DIFA, Università della Basilicata, Potenza, Italy \\ ${ }^{2}$ DiSGG, Università della Basilicata, Potenza, Italy \\ ${ }^{3}$ IMAA-CNR, Tito Scalo (PZ), Italy
}

Received: 21 July 2004 - Revised: 8 December 2004 - Accepted: 13 December 2004 - Published: 19 January 2005

Part of Special Issue "Precursory phenomena, seismic hazard evaluation and seismo-tectonic electromagnetic effects"

\begin{abstract}
Space-time TIR anomalies, observed from months to weeks before earthquake occurrence, have been suggested by several authors as pre-seismic signals. Up to now, such a claimed connection of TIR emission with seismic activity has been considered with some caution by scientific community mainly for the insufficiency of the validation data-sets and the scarce importance attached by those authors to other causes (e.g. meteorological) that, rather than seismic activity,

research (just started) of possible correlation among TIR anomalies and earthquake occurrence is concerned, such a result suggests that: a) in order to identify seismically unperturbed periods for confutation purposes, a magnitude threshold (at least) lower than 4 should be used; b) the proposed validation/confutation approach should be applied in lowseismicity areas in order to find suitably long seismically quiescent periods.
\end{abstract} could be responsible for the observed TIR signal fluctuations. A robust satellite data analysis technique (RAT) has been recently proposed which, thanks to a well-founded definition of TIR anomaly, seems to be able to identify anomalous spacetime TIR signal transients even in very variable observational (satellite view angle, land topography and coverage, etc.) and natural (e.g. meteorological) conditions.

Its possible application to satellite TIR surveys in seismically active regions has been already tested in the case of several earthquakes (Irpinia: 23 November 1980, Athens: 7 September 1999, Izmit: 17 August 1999) of magnitude higher than 5.5 by using a validation/confutation approach, devoted to verify the presence/absence of anomalous spacetime TIR transients in the presence/absence of seismic activity. In these cases, a magnitude threshold (generally $\mathrm{M}<5$ ) was arbitrarily chosen in order to identify seismically unperturbed periods for confutation purposes.

In this work, 9 medium-low magnitude $\left(4<\mathrm{M}_{b}<5.5\right)$ earthquakes which occurred in Greece and Turkey have been analyzed in order to verify if, even in these cases, anomalous TIR transients can be observed.

The analysis, which was performed using 8 years of Meteosat TIR observations, demonstrated that anomalous TIR transients can be observed even in the presence of mediumlow magnitude earthquakes $\left(4<\mathrm{M}_{b}<5.5\right)$. As far as the

Correspondence to: C. Filizzola

(filizzola@imaa.cnr.it)

\section{Introduction}

Several authors (see, for example, Qiang Zu-ji and Dian Chang-Gong, 1992; Qiang et al., 1991, 1997; Tronin, 1996, 2000; Tronin et al., 2002) have claimed a possible correlation between TIR $^{1}$ satellite anomalies, observed from months to weeks before an earthquake, and the occurrence of the seismic event itself. Such a claimed correlation has been considered, up to now, with some caution by scientific community mainly for the insufficiency of the validation data-sets and the scarce importance attached by those authors to other causes (e.g. meteorological) that, rather than seismic activity, could be responsible for the observed TIR signal fluctuations.

Moreover, the term anomaly is usually employed without any rigorous definition, but the actual detection of TIR anomalous signals is strictly linked to the possibility of discriminating between normal and anomalous signals.

As a matter of fact, a TIR satellite signal is the result of two main contributions at least: a natural one (e.g. connected to atmospheric transmittance, surface temperature, spectral emissivity and orography) and an observational one (e.g. depending on time of day, season and satellite view angles).

\footnotetext{
${ }^{1}$ Earth's thermally emitted radiation, which is measured from satellite in the Thermal Infrared $(8-14 \mu \mathrm{m})$ region of the electromagnetic spectrum, is usually referred to as TIR signal.
} 
Natural and observational conditions can be so highly variable, both in space and in time, that it cannot be excluded that a spatial-temporal anomaly, claimed to be in some connection with an earthquake, could be simulated or completely masked by normal natural/observational changes. In order to permit a suitable definition of a TIR anomaly, the two above-mentioned contributions to the signal should be preliminarily evaluated (in terms of signal-to-noise ratio, $\mathrm{S} / \mathrm{N}$ ) as natural/observational noise.

A statistically-based definition of TIR anomaly is at the root of the recent robust approach RAT (Robust AVHRR Techniques, Tramutoli, 1998), already applied to the analysis of space-time TIR anomalies in seismically active areas. It has been implemented, until now, by using satellite data acquired both from polar (AVHRR-NOAA ${ }^{2}$ ) and from geostationary ( $\mathrm{MVIRI}^{3}$-Meteosat) sensors and different seismically active areas have been studied following this approach: Southern Italy (Irpinia-Basilicata's earthquake, 23 November 1980, $\mathbf{M}_{s}=6.9, \mathbf{M}_{b}=6.0$, in Tramutoli et al., 2001; Di Bello et al., 2004), Greece (Athens's earthquake, 7 September 1999, $\mathbf{M}_{s}=5.9, \mathbf{M}_{b}=5.8$, in Filizzola et al., 2004) and Turkey (Izmit's earthquake, 17 August 1999, $\mathbf{M}_{s}=7.8$, $\mathbf{M}_{b}=6.8$, Tramutoli et al., 2004 ${ }^{4}$ ). In all cases, attention was put on the possible correlation between observed TIR anomalies and earthquakes with a magnitude greater than 5.5. In these cases, a magnitude threshold (generally $M<5$ ) was arbitrarily chosen in order to identify seismically unperturbed periods for confutation purposes.

In this paper, RAT is used once more for the study of the highly seismic Hellenic and Anatolian regions, but now focusing on the possibility that anomalous TIR signals may be observed even in the presence of earthquakes with mediumlow magnitude $\left(4 \leq \mathrm{M}_{b} \leq 5.5\right)$.

Unlike the greater part of the previous studies, this work relies upon the analysis of a longer historical data-set (8 years) of satellite (Meteosat) images and a greater number of seismic events ( 9 earthquakes) over a wide range of magnitude.

\section{RAT: a robust approach for thermal anomaly detec- tion}

The RAT (Robust AVHRR Techniques) approach was firstly proposed by Tramutoli (1998) as a general change-detection technique particularly suitable for natural (e.g. volcanoes,

\footnotetext{
${ }^{2}$ Advanced Very High Resolution Radiometer onboard NOAA (National Oceanographic and Atmospheric Administration) platforms.

${ }^{3}$ Meteosat Visible and Infra-Red Imager. MVIRI Infrared channel $(10.5-12.5 \mu \mathrm{m})$, characterized by a $5 \mathrm{~km}$ spatial resolution, was used in this work.

${ }^{4}$ Tramutoli, V., Cuomo, V., Filizzola, C., Pergola, N., and Pietrapertosa C.: Assessing the potential of thermal infrared satellite surveys for monitoring seismically active areas, The case of Kocaeli (Izmit) earthquake, 17 August 1999, Remote sensing of Environment (submitted), 2004.
}

floods and earthquakes) and environmental (e.g. forest fires) hazard monitoring.

As demonstrated in several works (see, for example, Cuomo et al., 2001, 2004; Di Bello et al., 2004; Bonfiglio et al., 2004; Lasaponara et al., 1998; Pergola et al., 1998, 2001, 2004a, 2004b), RAT turned out to be successful in various fields of application. Among them, it showed to be a valid tool for assessing the potential of TIR satellite surveys in seismically active regions. RAT actually provides a Robust Estimator of TIR Anomalies (RETIRA), being able to identify space-time TIR anomalies even in highly variable natural/observational conditions. It simply relies on the definition of the RETIRA index $\otimes\left(\boldsymbol{r}, t^{\prime}\right)$, computed pixel-bypixel on the image at hand:

$\otimes\left(\boldsymbol{r}, t^{\prime}\right) \equiv \frac{\left[\Delta T\left(\boldsymbol{r}, t^{\prime}\right)-<\Delta T(\boldsymbol{r})>\right]}{\sigma_{\Delta T}(\boldsymbol{r})}$,

where $\boldsymbol{r} \equiv(x, y)$ represents geographic coordinates of the image pixel center; $t^{\prime}$ is the acquisition time of the image at hand; $\Delta T\left(\boldsymbol{r}, t^{\prime}\right)$ is the difference between the current $\left(t=t^{\prime}\right)$ TIR signal value $T\left(\boldsymbol{r}, t^{\prime}\right)$ at location $\boldsymbol{r}$ and its spatial average $T\left(t^{\prime}\right)$, computed in place on the image at hand, considering cloud-free pixels only, all belonging to the same (land or sea) class in the investigated area. This means that $T\left(t^{\prime}\right)$ is computed considering only sea pixels if $\boldsymbol{r}$ is located over the sea and computed considering only land pixels if $\boldsymbol{r}$ is located over the land. It is worth noticing that the choice of such a differential variable $\Delta T\left(\boldsymbol{r}, t^{\prime}\right)$, instead of the simple $T\left(\boldsymbol{r}, t^{\prime}\right)$, is expected to reduce possible contributions (e.g. occasional warming) due to day-to-day and/or year-to-year climatological changes and/or season time-drifts; $\langle\Delta T(\boldsymbol{r})\rangle$ and $\sigma_{\Delta T}(\boldsymbol{r})$ are the time average and standard deviation values of $\Delta T(\boldsymbol{r}, t)$ computed on a homogeneous data-set, $t \in\{\mathcal{T}\}$, of cloud-free satellite records collected at location $\boldsymbol{r}$ within the same temporal slot (time of day) and period of year (month) of the image at hand $\left(t^{\prime} \in\{\mathcal{T}\}\right)$.

Both $<\Delta T(\boldsymbol{r})>$ and $\sigma_{\Delta T}(\boldsymbol{r})$ are computed, once and for all, for each location $\boldsymbol{r}$, processing several years of historical satellite records acquired under similar $\left(t, t^{\prime} \in\{\mathcal{T}\}\right)$ observational conditions. They are two reference images describing the normal behavior of the signal and of its variability at each location $\boldsymbol{r}$ in observational conditions as similar as possible to the ones of the image to be processed.

The $\otimes\left(\boldsymbol{r}, t^{\prime}\right)$ index gives the "llocal" ${ }^{5}$ excess of the current $\Delta T\left(\boldsymbol{r}, t^{\prime}\right)$ signal compared with its historical mean value and weighted by its historical variability at the considered location.

The excess, $\Delta T\left(\boldsymbol{r}, t^{\prime}\right)-<\Delta T(\boldsymbol{r})>$, then, represents the Signal $(S)$ to be investigated. It is always evaluated in comparison with the corresponding natural/observational Noise $(N)$, represented by $\sigma_{\Delta T}(\boldsymbol{r})$, which describes the "llocal" variability of $S$ including all (natural and observational, known and unknown) sources of its variability as historically

\footnotetext{
5 according to Tramutoli (1998) the double $l$ is used hereafter to specify not only a certain place $\boldsymbol{r}^{\prime}$ but also a certain time $t^{\prime}$.
} 


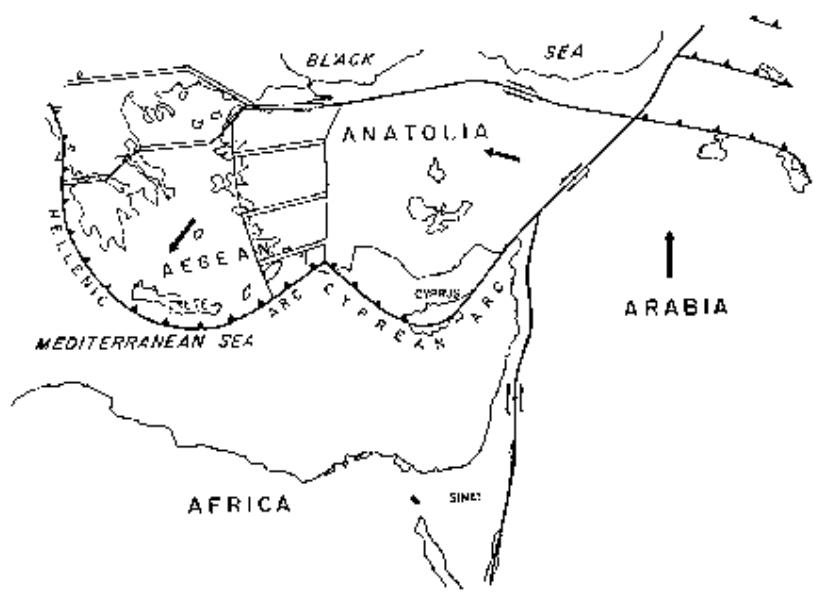

Fig. 1. Schematic map of the main tectonic systems in the Aegean and Anatolian Regions (Rotstein e Kafka, 1982).

observed at the same site under similar observational conditions. This way, such an approach allows us to evaluate in terms of $S / N$ ratio the relative importance of a measured TIR signal. Finally, it is not meaningless to emphasize that larger $\sigma_{\Delta T}(\boldsymbol{r})$ values determine lower $\otimes\left(\boldsymbol{r}, t^{\prime}\right)$ values, protecting the index against false alarm proliferation (robustness).

\section{Test cases}

In this paper, the sensitivity of the RAT technique has been evaluated in the case of 9 earthquakes, characterized by magnitudes within the range $4 \div 5.5$, which occurred in an area of about 1.5 million $\mathrm{km}^{2}$ (latitude: $33^{\circ} \div 45^{\circ} \mathrm{N}$; longitude: $19^{\circ} \div 48^{\circ} \mathrm{E}$ ), mainly consisting of the Aegean and Anatolian regions.

The area under study is characterized by high (both in frequency and in intensity) seismicity, which is particularly concentrated along the Hellenic Arc subduction zone, throughout the Aegean Sea, along the North and East Anatolian faults, the Cyprian Arc and the Dead Sea fault (Fig. 1). These areas are geographically adjacent, but their geo-tectonic settings can be very different and, as a direct consequence, the produced earthquakes may show different characteristics from one area to another. For example, the North Anatolian fault (responsible for the disastrous earthquake in Izmit), running from one end to the other of Northern Turkey, is a right strike-slip fault which splits up into transtensive structures in its more western part and crosses a left transpressive fault (the East Anatolian Fault) in its more eastern part.

Within this large area, five test cases (single earthquakes or series of seismic events, which occurred at short time distances) have been considered for validation/confutation purposes:

a) the series of three seismic events which started on 28 May (Patras) and carried on with the earthquakes on 29 May (Cyprian region) and 3 June (Crete) 1995;

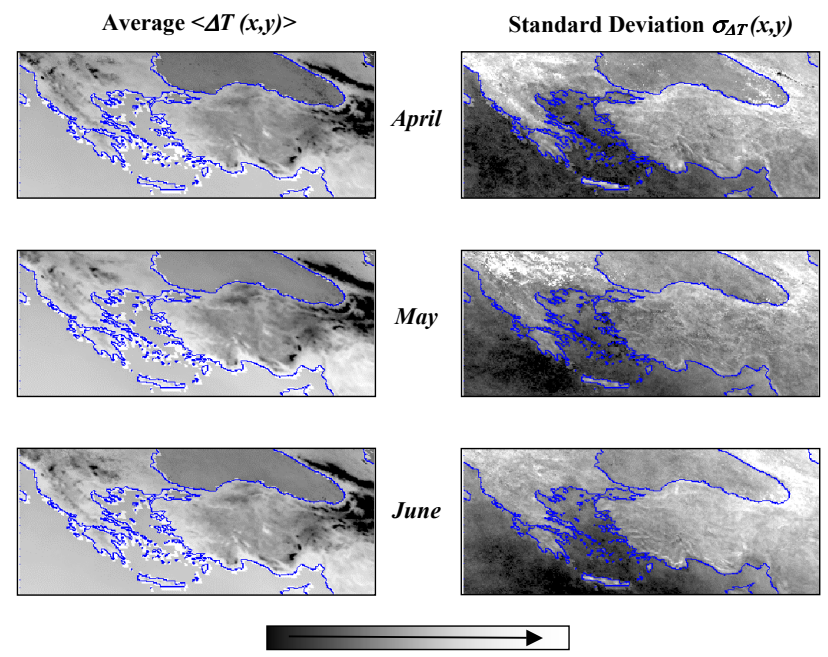

Fig. 2. Reference Fields (average and standard deviation) for April, May and June. They are computed on the basis of monthly data sets, consisting of Meteosat TIR images acquired at 24:00 GMT from 1992 to 1999 (copyright 2004 EUMETSAT).

b) Crete's earthquake which occurred on 18 June 1995;

c) Erzurum's earthquake which involved Turkey on 4 May 1996;

d) the series of earthquakes which occurred in the Ionian Sea (13 June 1996), Patras (16 June 1996) and Crete (17 June 1996);

e) Isparta's seismic event that shook Turkey on 29 June 1996.

\subsection{Validation}

All Meteosat TIR images acquired in the same time slot (23:30-24:00 GMT), during the same period of the year (April, May, June) from 1992 to 1999 were collected in monthly data-sets and used to build two reference fields (average and standard deviation) for each considered month (Fig. 2). Starting from these fields, the RETIRA $\otimes\left(\boldsymbol{r}, t^{\prime}\right)$ index was computed for each image of the above-mentioned data-sets and the space-time distribution of TIR anomalies, associated to pixels having $\otimes\left(\boldsymbol{r}, t^{\prime}\right)$ greater than 3 (i.e. $\left.\Delta T\left(\boldsymbol{r}, t^{\prime}\right)-<\Delta T \boldsymbol{r}\right)>$ excess greater than $\left.3 \sigma_{\Delta T}(\boldsymbol{r})\right)$, was analyzed. The results concerning the periods affected by the studied seismic events are shown and described in the following lines.

a) In the first test case, seismic events, characterized by medium-low magnitude, which occurred in a short timeinterval have been analyzed:

- Patras (lat. $38.41^{\circ} \mathrm{N}$, lon. $22.17^{\circ} \mathrm{E}$, depth $28.0 \mathrm{~km}$ ), 28 May $1995, \mathrm{M}_{b}=4.7$

- Cyprus (lat. $34.99^{\circ} \mathrm{N}$, lon. $32.19^{\circ} \mathrm{E}$, depth $28.9 \mathrm{~km}$ ), 29 May $1995, \mathrm{M}_{b}=5.3$; 

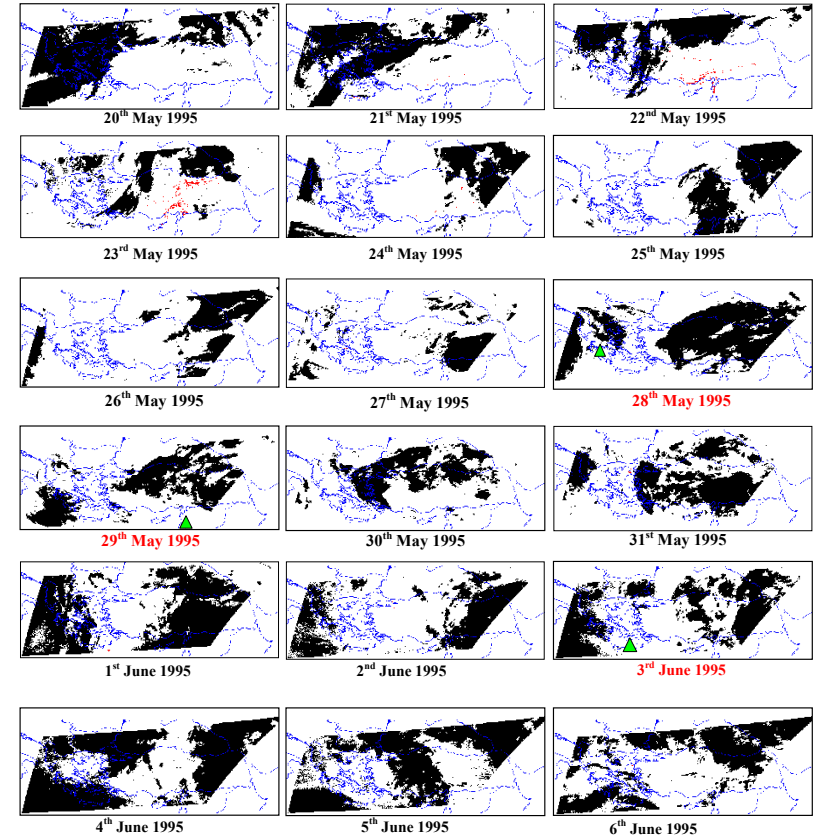

$\triangle$ Epicentre $\square \otimes(x, y, t)>3 \quad$ Cloud
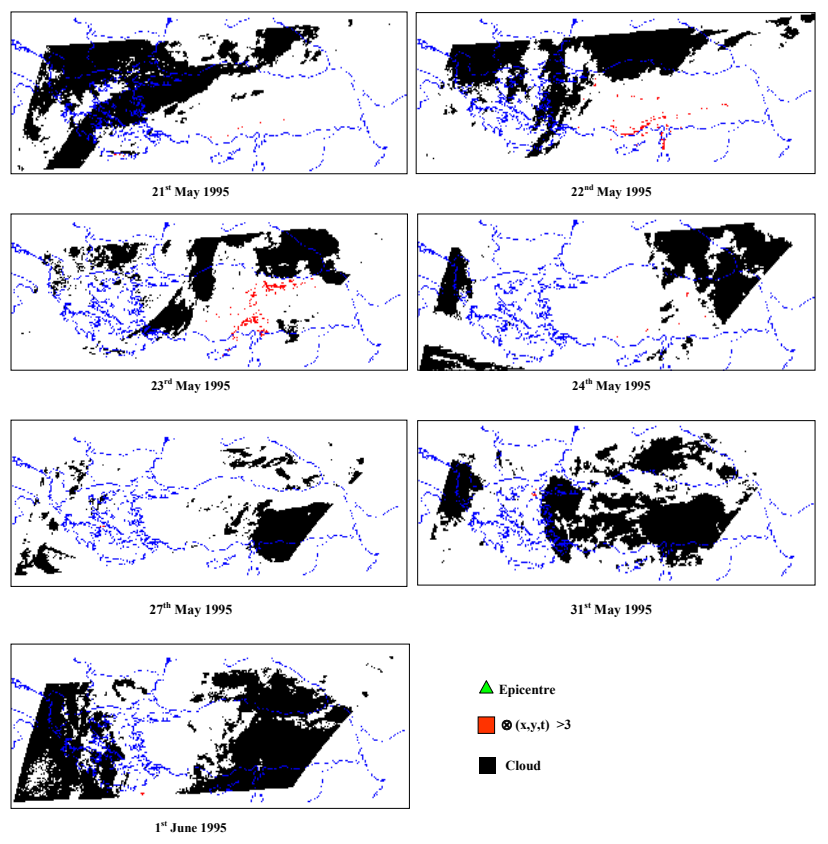

Fig. 3. (a) Results of the $\otimes(x, y, t)$ computation from the preseismic to the post-seismic period, considering a series of three earthquakes which involved the studied area between May and June 1995 (see text). All images come from the processing of Meteosat TIR observations collected at 24:00 GMT (copyright 2004 EUMETSAT). (b) Enlarged images of the sequence (a) showing RETIRA values greater than 3 .

- Crete (lat. $34.23^{\circ} \mathrm{N}$, lon. $25.51^{\circ} \mathrm{E}$, depth $40.0 \mathrm{~km}$ ), 3 June 1995, $\mathrm{M}_{b}=4.2$.

Looking at the images in Fig. 3a (and even better in Fig. 3b), the first TIR anomalous signals can be observed on 21 May over Crete and southern Turkish coasts (Central Taurus). They become wider and wider (particularly towards NW) in the following days, reaching their maximum extension (until Northern Turkish coasts) on 23 May. Starting from 24 May, such anomalies decrease until they disappear during and after the occurrence of the seismic events. It should be noted that the spatial distribution of the observed TIR anomalies (particularly on 23 May) seems to follow the principal tectonic structures of the area, as shown in Fig. 4.

b) The second test case concerns the seismic event which occurred in Crete (lat. $34.41^{\circ} \mathrm{N}$, lon. $25.41^{\circ} \mathrm{E}$, depth $\left.29.6 \mathrm{~km}, \mathrm{M}_{b}=4.9\right)$ on 18 June 1995 , along the Hellenic Arc subduction zone. Some TIR anomalies (Fig. 5) appear on 10 June over the Mediterranean Sea (near the south-east coasts of Crete) and increase their spatial extension until their maximum on 13 June (five days before the earthquake). Like the first test case, the observed TIR anomalies decrease until they disappear during and after the seismic event.

c) The Erzurum's earthquake has been studied as the third test case. It occurred (4 May 1996, lat. $39.02^{\circ} \mathrm{N}$, lon. $28.03^{\circ} \mathrm{E}$, depth $10.0 \mathrm{~km}, \mathrm{M}_{b}=4.2$ ) in a tectonically complex area where two mountain chains join (east of the North Anatolian fault). Three days before the occurrence of the earthquake, several TIR anomalies are detected along the North Anatolian fault, as shown in the sequence of images in Fig. 6 and more clearly in Fig. 7. Again, during and after the seismic event, no TIR anomalous signals are detected.

d) A further test case is represented by a series of three seismic events that involved:

- The Ionian Sea (lat. $37.56^{\circ} \mathrm{N}$, lon. $21.10^{\circ} \mathrm{E}$, depth $29.4 \mathrm{~km}$ ), 13 June 1996, $\mathrm{M}_{b}=4.2$;

- Patras (lat. $38.41^{\circ} \mathrm{N}$, lon. $22.10^{\circ} \mathrm{E}$, depth 14.9), 16 June 1996, $\mathrm{M}_{b}=4.3$;

- Crete (lat $34.73^{\circ} \mathrm{N}$, lon. $25.36^{\circ} \mathrm{E}$, depth 1.9), 17 June $1996, \mathrm{M}_{b}=4.3$.

Such events have been grouped as a unique series because they occurred both spatially and temporarily at very short distances (Fig. 8b). Figure 8a shows the presence of very isolated TIR anomalous signals from 7 to 11 June.

Despite the very limited extension of TIR anomaly patterns (Fig. 8a), their spatial distribution along the main tectonic structures of the area (Fig. 8c) should be noted. In particular, the areal distribution of the anomalies appears to delineate the Hellenic Arc.

Small (up to $100 \mathrm{~m}^{2}$ ) forest fires can produce significant IR signal variations particularly in the MIR (3-4 micron) region and, to a lesser extent, in the TIR (10-12 micron) region. The possible relation between observed TIR anomalies and forest fires (very frequent in Greece during the warmer season) has been closely (by using higher spatial resolution MIR and TIR AVHRR observations) investigated only in the case of isolated (few pixels) spatially persistent TIR anomalies. As a matter of fact, considering the $5 \mathrm{~km}$ spatial resolution of Meteosat TIR imagery it is difficult to associate to forest fires 


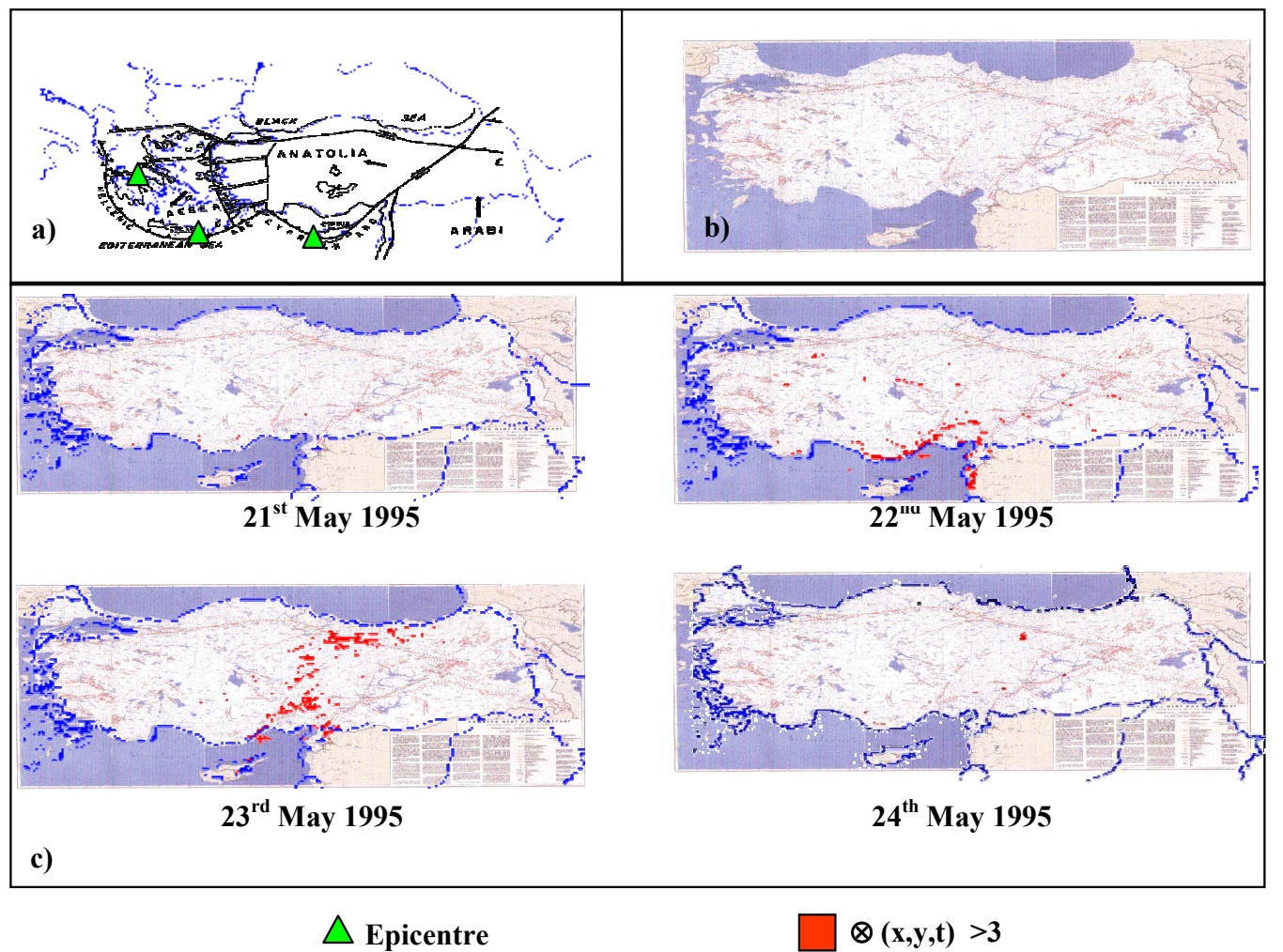

Fig. 4. (a) Schematic map of the principal tectonic structures of the Eastern Mediterranean Sea (Rodstein and Kafka, 1982; modified); (b) Active fault map of Turkey (produced by Geological Research Department MTA - MADEN TETKIK ARAMA - Ankara); (c) TIR anomalies overlapped the active fault map: the spatial distribution of TIR anomalies seems to follow the tectonic structures of the region.

thermal anomalies of bigger extension or moving, as is our case, for tens of kilometres per days. This was the case only of the sequence described in Fig. 8, where a spatially isolated and persistent thermal anomaly is present in the upper part of 7 June ( 3 pixels) and 10 June (4 pixels) images. The analysis performed using NOAA AVHRR imagery (MIR and TIR channels) permitted us to exclude the forest fire hypothesis. RETIRA index (which is, by construction, independent from thermal gradients associated to orography) identified, in this case, only few TIR anomalies within a wide area (about $800 \mathrm{~km}^{2}$ ) corresponding to an orography depression showing from 7 June until 10 June normally (without punctual excesses associable to fires) high (brightness temperature up to $295 \mathrm{~K})$ MIR and TIR signals.

e) Concerning the Isparta's earthquake (29 June 1996, lat. $38.02^{\circ} \mathrm{N}$, lon. $30.06^{\circ} \mathrm{E}$, depth $30.7 \mathrm{~km}, \mathrm{M}_{b}=5.1$ ), starting from 24 June (Fig. 9), anomalous signals are detected along Turkish coasts (Central Taurus) until 25 June. No anomalous values are observable after the earthquake. TIR observed anomalies, earthquake epicentre and the active tectonic structures are reported in Fig. 10.

It is worth noticing that TIR anomalies are clearly present in the images of 27 and 28 affecting the Eastern part of Turkey and Black Sea where earthquakes with medium-low magnitude $\left(4 \leq \mathrm{M}_{b} \leq 4.7\right)$ occurred from the end of June to the beginning of July 1996 (Fig. 11).

\subsection{Confutation}

Confutation has been performed in order to verify the absence of TIR anomalies in seismically "unperturbed" (hereafter is used to indicate absence of earthquakes with magnitude greater than - or equal to -4 ) periods. The confutation process was particularly difficult for a highly seismic active area like the Aegean-Anatolian region, which is a part of the world that is never seismically at rest. Due to the high seismicity of such a wide area, "unperturbed" periods to be used for confutation purposes revealed to be rare and very short.

From the consulted seismic catalogs (CNSS, IRIS, ANSS), the time-interval from 3 to 13 June 1998 turns out not to be affected by earthquakes with a magnitude greater than (or equal to) 4.

The monthly data-sets of Meteosat TIR images, used for validation purposes, were also employed to analyze seismically unperturbed periods. The sequence of images shown in Fig. 12 concerns the results of a few days prior to the abovementioned unperturbed time-interval: it is quite evident that no TIR anomaly is identified during these days.

Confutation phase was performed also for the post-seismic period of the studied earthquakes, if aftershocks with magnitude greater than (or equal to) 4 did not occur. In all these cases, no TIR anomalies were detected, as already shown in Figs. 3, 5, 6 and 8 . 


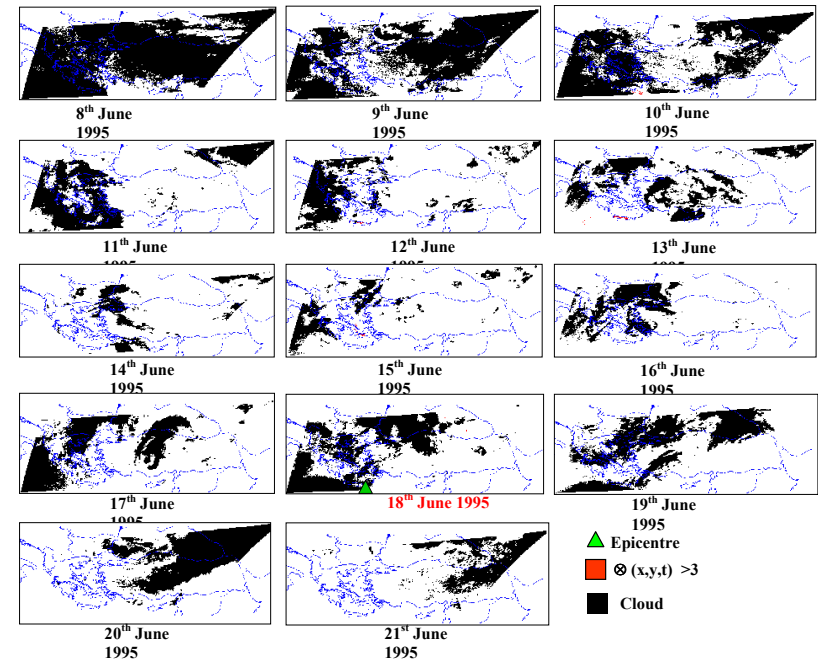

Fig. 5. Results of the $\otimes(x, y, t)$ computation for the period straddling Crete's earthquake (see text). All images come from the processing of Meteosat TIR observations collected at 24:00 GMT (copyright 2004 EUMETSAT).

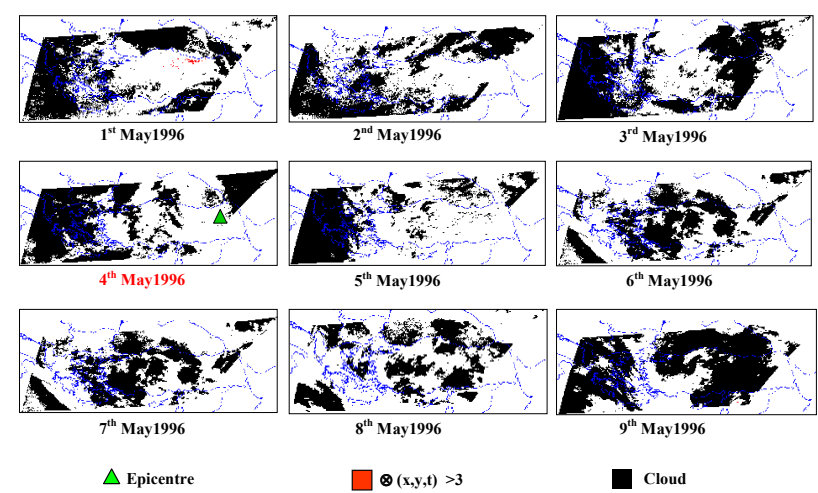

Fig. 6. Results of the $\otimes(x, y, t)$ computation for the period straddling Erzurum's earthquake (see text). All images come from the processing of Meteosat TIR observations collected at 24:00 GMT (copyright 2004 EUMETSAT).

\section{Conclusions and future improvements}

In order to verify, by a validation/confutation approach, the presence/absence of anomalous space-time TIR transients in the presence/absence of seismic activity, previous studies (Tramutoli et al., 2001; Di Bello et al., 2004; Filizzola et al., 2004; Tramutoli et al., $2004^{6}$ ) used an arbitrarily chosen magnitude threshold (generally $\mathrm{M}<5$ ) to identify seismically unperturbed conditions for confutation purposes.

\footnotetext{
${ }^{6}$ Tramutoli, V., Cuomo, V., Filizzola, C., Pergola, N., and Pietrapertosa C.: Assessing the potential of thermal infrared satellite surveys for monitoring seismically active areas, The case of Kocaeli (Izmit) earthquake, 17 August 1999, Remote sensing of Environment (submitted), 2004.
}

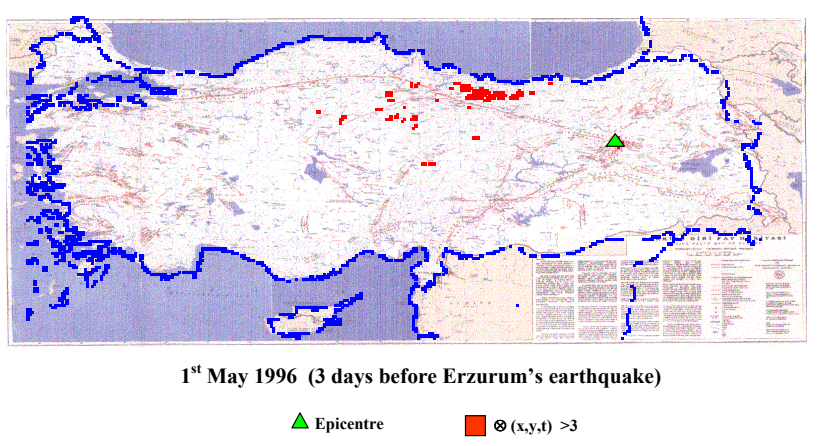

Fig. 7. TIR anomalies (in red) observed on the Meteosat image of 1 May 1996, i.e. three days before the occurrence of Erzurum's seismic event. They overlap the principal tectonic lineaments of the area.

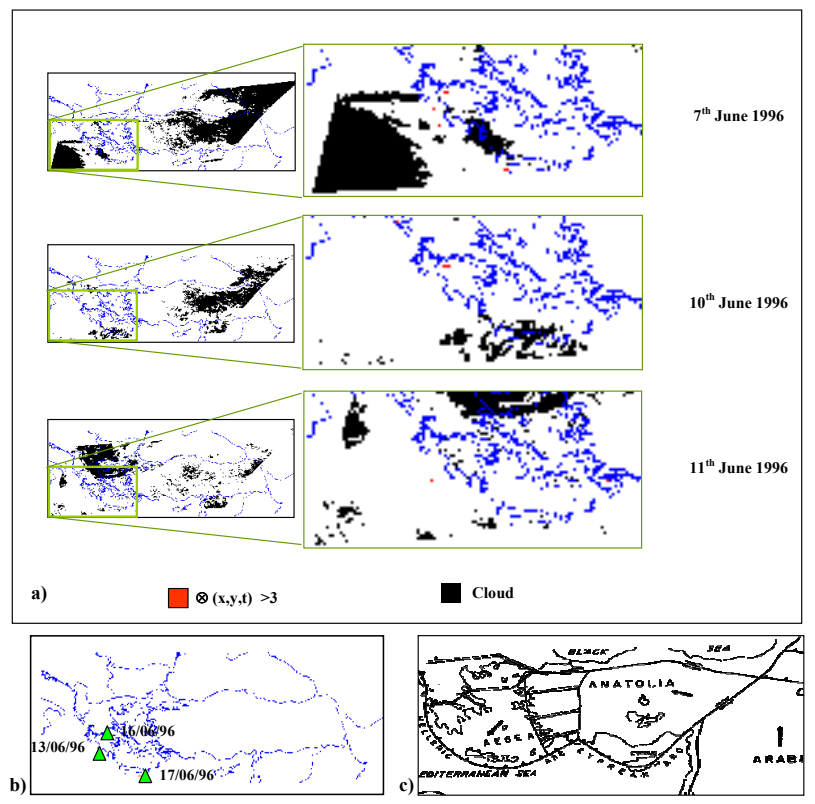

Fig. 8. (a) Results of $\otimes(x, y, t)$ computation from the pre-seismic to the post-seismic period, considering a series of three earthquakes which involved the studied area during June 1996 (see text). Only images showing TIR anomalous values are presented, together with a zoom of the area affected by these anomalies; (b) epicentre position (green triangles) and occurrence of the three earthquakes of the considered series; (c) the principal tectonic structures of the area. All images come from the processing of Meteosat TIR observations collected at 24:00 GMT (copyright 2004 EUMETSAT).

In this paper, using the same (RAT, Tramutoli, 1998) approach, and 8 years of Meteosat TIR observations, the possible occurrence of anomalous space-time TIR transients has been investigated in the case of 9 seismic events $\left(4 \leq \mathrm{M}_{b} \leq 5.5\right)$ which occurred in the Aegean and Anatolian regions in 1995 and 1996.

The analysis showed that:

1. TIR anomalies identified by RETIRA index values $\otimes\left(\boldsymbol{r}, t^{\prime}\right)>3$ (i.e. signal excesses $\Delta T\left(\boldsymbol{r}, t^{\prime}\right)-<\Delta T(\boldsymbol{r})>$ 


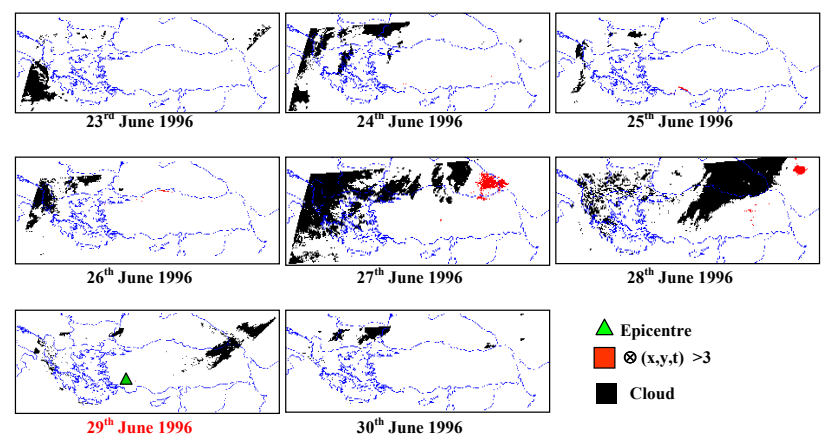

Fig. 9. Results of the $\otimes(x, y, t)$ computation for days straddling Isparta's earthquake (see text). All images come from the processing of Meteosat TIR observations collected at 24:00 GMT (copyright 2004 EUMETSAT).

greater than $3 \sigma_{\Delta T}(\boldsymbol{r})$ ), occurred very rarely (in the space-time domain) during the 8 year long period of observations. This confirmed the capability of the proposed approach to filter-out most of the variable contributions to the TIR signal originated by the normal space-time variability of several natural/observational factors;

2. anomalous space-time TIR transients (of minor extension but with similar intensity, compared with the ones observed in the above mentioned studies for earthquakes with $\mathrm{M}>5.5$ ) have been observed even in the presence of medium-low magnitude $\left(4<\mathrm{M}_{b}<5.5\right)$ earthquakes;

3. in all 9 test cases, a similar trend was observed:

- a first appearance of anomalous TIR signals a few days before the occurrence of the earthquake

- an increase in the spatial extension of TIR anomaly up to a maximum, followed by a more or less quick decrease until a complete disappearance during and after the earthquake

4. whenever seismically unperturbed (i.e. no earthquakes with $\mathrm{M}>4$ ) periods were identified no TIR anomalies were observed.

As far as the research (just at its start) of possible correlations (still far to be demonstrated and out of the scope of this paper) between TIR anomalies and earthquake occurrence is concerned, our results suggest that:

1. in order to identify seismically unperturbed periods for confutation purposes, a magnitude threshold (at least) lower than 4 should be used;

2. the proposed validation/confutation approach should be applied in low-seismicity areas in order to find suitably long seismically quiescent periods.

As a matter of fact, the present study revealed the difficulty to perform the confutation step (which is not less, or even
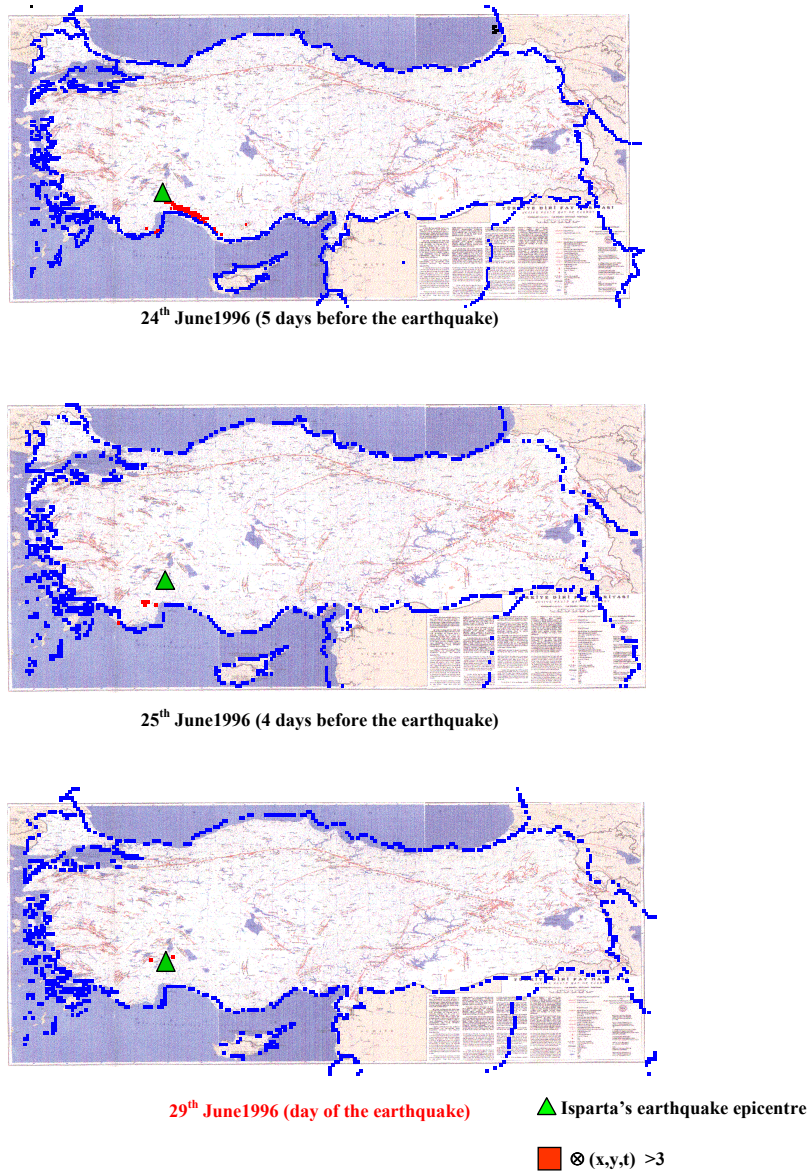

Fig. 10. A synoptic view of the TIR observed anomalies, the earthquake epicentre and the active fault map of Turkey.

more important, than the validation process) in areas with high seismicity because, mainly in this case, to find long enough seismically unperturbed periods is not always possible.

By the other side, the observation of TIR anomalies at a $\mathrm{S} / \mathrm{N}$ level greater than 3 , even in the presence of earthquakes, characterized by medium-low intensity, seems to suggest that there is no direct correlation between TIR anomaly intensity and earthquake magnitude.

This aspect could be better understood, for example, by analyzing a unique seismic sequence (rather than studying different spatially scattered earthquakes) generated in the same area (i.e. the same tectonic structure) in order to minimize possible site effects related to the local geo-tectonic setting. 


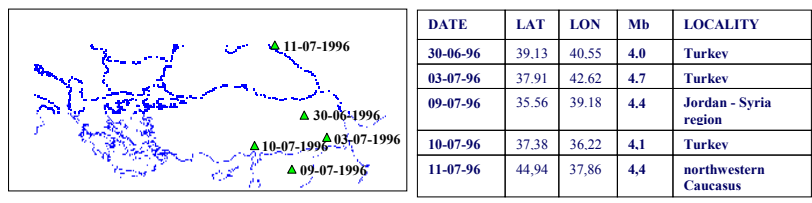

Fig. 11. Epicentre position and occurrence of seismic events from the end of June to the beginning of July 1996. Compare them with TIR anomalies over the images of 27 and 28 June in Fig. 10.

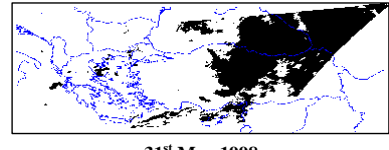

$31^{\text {st }}$ May 1998

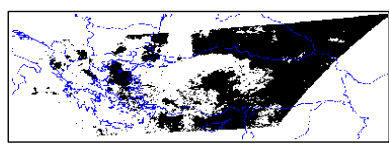

$2^{\text {nd }}$ June 1998

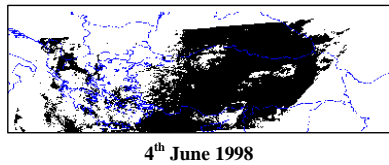

$4^{\text {th }}$ June 1998

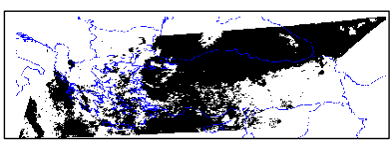

$6^{\text {th }}$ June 1998

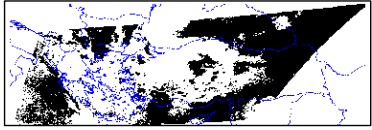

$1^{\text {st }}$ June 1998

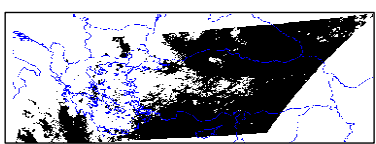

$3^{\text {rd }}$ June 1998

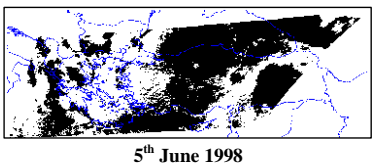

$5^{\text {th }}$ June 1998

Cloud
Fig. 12. Results of the $\otimes(x, y, t)$ computation for an unperturbed period (no earthquake with $M>4$ occurred): no TIR anomaly is detected.

Acknowledgements. This work has been carried out within the framework of the SEISMASS (SEISmic area Monitoring by Advanced Satellite Systems) project funded by the Italian Space Agency (contract N.I/R/13/02). The Authors thanks A. Tzanis for closely and accurately reviewing the earlier version of the manuscript, improving its clarity and completeness.

Edited by: P. F. Biagi

Reviewed by: A. Tzanis and another referee

\section{References}

ANSS: Advanced National Seismic System, http://www.anss.org, 2002.

Bonfiglio, A., Macchiato, M., Pergola, N., Pietrapertosa, C., and Tramutoli, V.: AVHRR Automated detection of volcanic clouds, Int. J. Rem. Sens., in press, 2005.

CNSS: Council of the National Seismic system, http://www.cnss. org, 2002.

Cuomo, V., Filizzola, C., Pergola, N., Pietrapertosa, C., and Tramutoli, V.:A self-sufficient approach for GERB cloudy radiance detection, Special Issue on "Clouds and Radiation", Atmos. Res., 72, 1-4, 39-56, 2004.

Cuomo, V., Lasaponara, R., and Tramutoli, V.: Evaluation of a new satellite-based method for forest fire detection, Int. J. Rem. Sens., 22, 9, 1799-1826, 2001.
Di Bello, G., Filizzola, C., Lacava, T., Marchese, F., Pergola, N., Pietrapertosa, C., Piscitelli, S., Scaffidi, I., and Tramutoli, V.: Robust Satellite Techniques for Volcanic and Seismic Hazards Monitoring, Annals of Geophysics, 47, 1, 49-64, 2004.

Filizzola, C., Pergola, N., Pietrapertosa, C., and Tramutoli, V.: Robust satellite techniques for seismically active areas monitoring: a sensitivity analysis on September 7th 1999 Athens's earthquake, Special issue on Seismo Electromagnetics and Related Phenomena, Phys. Chem. Earth, 29, 4-9, 517-527, 2004.

IRIS: Incorporated Research Institutions For Seismology, http:// www.iris.edu.

Lasaponara, R., Tramutoli, V., and Cuomo, V.: Fire-detection by AVHRR: toward a new generalized approach for operational monitoring, Remote Sensing for Agriculture, Ecosystems and Hydrology, edited by Engman, E. T., Proceedings of SPIE, 3499, 348-358, 1998.

Pergola, N., Pietrapertosa, C., Lacava, T., and Tramutoli, V.: Robust Satellite Techniques for Monitoring Volcanic Eruptions, Annals of Geophysics, 44, 2, 167-177, 2001.

Pergola, N., Tramutoli, V., and Pietrapertosa, C.: Satellite Remote Sensing of Volcanic Aerosols: a new, AVHRR-based, approach, Satellite Remote Sensing of Clouds and the Atmosphere III, edited by Russel, J. E., Proceedings of SPIE, 3495, 188-197, 1998.

Pergola, N., Tramutoli, V., Scaffidi, I., Lacava, T. and Marchese, F.: Improving volcanic ash clouds detection by a robust satellite technique, Remote Sensing of Environment, 90, 1, 1-22, 2004a.

Pergola, N., Marchese, F., and Tramutoli, V.: Automatic detection of thermal features of active volcanoes by means of infrared AVHRR records, Remote Sensing of Environment, 93, 311-327, 2004b.

Qiang Z.-j. and Dian C.-G.: Satellite Thermal Infrared Impending Temperature Increase Precursor of Gonghe Earthquake of Magnitude 7.0, Qinghai Province, Geoscience, 6, 3, 297-300, 1992.

Qiang, Z.-j., Xu, X.-D., and Dian, C.-G.: Thermal infrared anomaly precursor of impending earthquakes, Chinese Science Bulletin, 36, 4, 319-323, 1991.

Qiang, Z.-j., Xu, X.-D., and Dian, C.-G.: Thermal infrared anomaly precursor of impending earthquakes, Pure and Applied Geophysics, 149, 159-171, 1997.

Rotstein, Y. and Kafka, A. L.: Seismotectonics of the southern boundary of Anatolia, Eastern Mediterranean Region: subduction, collision and arc jumping, J. Geophys. Res., 87, 7694-7706, 1982.

Tramutoli, V.: Robust AVHRR Techniques (RAT for Environmental Monitoring: theory and applications, Earth Surface Remote Sensing II, edited by Cecchi, G. and Zilioli, E., Proceedings of SPIE, 3496, 101-113, 1998.

Tramutoli, V., Di Bello, G., Pergola, N., Piscitelli, S.: Robust satellite techniques for remote sensing of seismically active areas, Annali di Geofisica, 44 (2), 295-312, 2001.

Tronin, A. A.: Satellite thermal survey - a new tool for the study of seismoactive regions, Int. J. Remote Sensing, 41, 8, 1439-1455, 1996.

Tronin, A. A.: Thermal IR satellite sensor data application for earthquake research in China, Int. J. Remote Sensing, 21, 16, 31693177, 2000.

Tronin, A. A., Hayakawa, M., and Molchanov, O. A.: Thermal IR satellite data application for earthquake research in Japan and China, J. Geodyn., 33, 4-5, 519-534, 2002. 